\title{
Scintigraphic Patterns of Indium-111 Oxine-Labeled White Blood Cell Imaging of Gram-Negative versus Gram-Positive Vertebral Osteomyelitis
}

\author{
Abdelhamid H. Elgazzar ${ }^{a}$ Shorouk Dannoon ${ }^{a}$ Ismet Sarikaya ${ }^{a}$ \\ Medhat Farghalia ${ }^{a}$ Thamradeen A. Junaid ${ }^{b}$ \\ Departments of a Nuclear Medicine and ${ }^{b}$ Pathology, Faculty of Medicine, Kuwait University, Kuwait, Kuwait
}

\section{Significance of the Study}

- In this study of induced vertebral osteomyelitis in rabbits, while the majority of the gram-positive infections showed accumulation of ${ }^{111}$ In-oxine-labeled white blood cells ( ${ }^{111} \mathrm{In}$-WBC), most of the gramnegative infections did not show ${ }^{111} \mathrm{In}-\mathrm{WBC}$ uptake. The nonaccumulation of ${ }^{111} \mathrm{In}-\mathrm{WBC}$ at the gramnegative site of infection could be due to the secretion of antichemotactic factors by gram-negative organisms. Hence, the cold spots observed on vertebral osteomyelitis should alert physicians to the possibility of the presence of gram-negative organism as a cause of the infection.

\section{Keywords}

Vertebral osteomyelitis · Scintigraphy $\cdot{ }^{111}$ In-oxine-labeled white blood cell imaging · Gram-negative organisms · Rabbit

\begin{abstract}
Objective: The goal of the study was to investigate whether or not gram-negative organisms that secrete antichemotactic factors cause the nonaccumulation pattern of ${ }^{111}$ In-oxinelabeled white blood cell ( ${ }^{111}$ In-WBC) scans. Materials and Methods: Staphylococcus aureus (gram-positive) (group 1) was injected into 25 rabbits and Escherichia coli (gram-negative) (group 2) into another 25 to induce infection in the lumbar vertebrae or left thigh bone (femur). Sixteen successfully infected and surviving rabbits from each group were
\end{abstract}

\section{KARGER}

E-Mail karger@karger.com www.karger.com/mpp
(C) 2017 The Author(s)

Published by S. Karger AG, Basel

\section{Karger \\ Open access}

This is an Open Access article licensed under the Creative Commons Attribution-NonCommercial-4.0 International License (CC BY-NC) (http://www.karger.com/Services/OpenAccessLicense), applicable to the online version of the article only. Usage and distribution for commercial purposes requires written permission. used for imaging and analysis. Of the 16 rabbits, each group included 8 with vertebral infection and 8 with femur infection. For imaging, each rabbit was injected intravenously with $11.1 \mathrm{MBq}(300 \mu \mathrm{Ci})^{111} \mathrm{In}-\mathrm{WBC}$, and images were acquired $24 \mathrm{~h}$ later. Microscopic histopathology was performed after decalcification to confirm osteomyelitis. Results: The ${ }^{111}$ In-WBC accumulation was observed in 7 (87.5\%) of the 8 rabbits infected with $S$. aureus in the vertebrae and thigh bone. Of the rabbits infected with the gram-negative vertebrae, 1 (12.5\%) showed little accumulation of ${ }^{111}$ In-WBC. Of the 8 rabbits with gram-negative-infected femurs, 1 had high accumulation and another had low accumulation of ${ }^{111}$ In-WBC, while the rest did not show any uptake. Osteomyelitis was confirmed by histopathology in all the successfully infected rabbits used for imaging. Conclusion: In the major-

Dr. Abdelhamid H. Elgazzar

Department of Nuclear Medicine, Faculty of Medicine Kuwait University, PO Box 24923

Safat, Kuwait 13110 (Kuwait)

E-Mail aelgazzar49@ hotmail.com 
ity of the gram-positive-infected rabbit vertebrae there was high accumulation of ${ }^{111}$ In-WBC. However, no accumulation of ${ }^{111}$ In-WBC was observed in most of the vertebrae infected with gram-negative organisms, which release antichemotactic factors that prevent adequate accumulation of WBC at the infected area.

(c) 2017 The Author(s)

Published by S. Karger AG, Basel

\section{Introduction}

Spinal infections are increasing in incidence, accounting for $2-7 \%$ of all musculoskeletal infections [1]. Standard radiographs are neither sensitive nor specific for the diagnosis of the relatively common condition [2]. Bone scanning is sensitive but is not specific [2]. Radiolabeled white blood cell (WBC) scintigraphy is accurate and specific, and the method of choice for diagnosing and localizing acute skeletal infections [3]. However, radiolabeled WBC scanning using either ${ }^{111} \mathrm{In}$-oxine or ${ }^{99 \mathrm{~m} \mathrm{Tc} \text { - }}$ HMPAO was shown to be neither sensitive nor specific for vertebral osteomyelitis [4-6]. This low sensitivity was due to different patterns of uptake - normal, low, or high - in cases of proven vertebral osteomyelitis, as demonstrated in several studies $[7,8]$. Normal, low, and high uptake of ${ }^{111}$ In-oxine-labeled WBC $\left({ }^{111} \mathrm{In}\right.$-WBC) was reported in patients by Palestro et al. [7], who studied 71 patients with suspected vertebral osteomyelitis and found that ${ }^{111}$ In-WBC scintigraphy showed normal uptake in 2, increased in 11, and decreased in 15 cases with proven osteomyelitis [7]. Increased uptake was associated with a high specificity of $98 \%$, but low sensitivity (39\%). The photopenic pattern was neither sensitive (54\%) nor specific (52\%) for osteomyelitis. Whalen et al. [8] reported a sensitivity of $17 \%$, a specificity of $100 \%$, and an accuracy of $31 \%$ for ${ }^{111}$ In-WBC imaging. The authors found photon-deficient areas at the sites of proven osteomyelitis in 15 of 18 patients, and they were included in the false-negative scans. Using ${ }^{99 \mathrm{~m}} \mathrm{Tc}-\mathrm{HMPAO}$-labeled WBC, Ruther et al. [9] reported 5 cases of proven vertebral osteomyelitis that were all missed. Hovi et al. [10] also reported 3 cases of histologically proven infection detected by magnetic resonance imaging but none by ${ }^{99 \mathrm{~m}} \mathrm{Tc}-\mathrm{HMPAO}-\mathrm{la}$ beled WBC studies.

Because the diagnosis of vertebral osteomyelitis is often delayed, most infections become chronic in nature, which could in part explain the low sensitivity of ${ }^{111} \mathrm{In}$ WBC in their diagnosis, as in decreased (photopenic) areas on ${ }^{111}$ In-WBC imaging of proven vertebral osteomyelitis. An alternative probable explanation could be the very high pressure in the disk with infection, and hence the granulocytes could not migrate to the focal area during the available time for imaging [11]. Fernandez-Ulloa et al. [12] previously reported 5 cases of vertebral osteomyelitis secondary to gram-negative organisms (Pseudomonas aeruginosa and Klebsiella pneumoniae); 4 showed areas of decreased uptake (photon deficient) on ${ }^{111} \mathrm{In}$ WBC scans, suggesting that these organisms could have prevented adequate accumulation of labeled cells at the site of infection.

Hence, the objective of this study was to investigate whether gram-positive or gram-negative bacterial infection causes the uptake of ${ }^{111} \mathrm{In}$-WBC at the site of osteomyelitis in the vertebrae or thigh bone.

\section{Materials and Methods}

\section{Small Animals}

Fifty adult male New Zealand white rabbits weighing approximately $3.5 \mathrm{~kg}$ divided into 2 groups of 25 each were used in this study. Staphylococcus aureus (group 1) (gram-positive bacteria) was injected into 25 rabbits and Escherichia coli (group 2) (gramnegative bacteria) to the remaining 25 to induce infection in the lumbar vertebrae or left thigh bone. Sixteen successfully infected and surviving rabbits from each group were used for the rest of the study. Each group included 8 rabbits with vertebral infection and 8 with femur infection. Experiments were performed in accordance with guidelines approved by the Institutional Animal Care and Use Committee of Kuwait University. Osteomyelitis induction via a spine implant infection model was performed, as previously described by Poelstra et al. [13]. Briefly, the marginal veins in rabbit ears were connected to butterfly needles. The rabbits were anesthetized with sodium pentobarbital $(40 \mathrm{mg} / \mathrm{kg}$, i.v.). The implantation sites (the lumbar vertebrae or left thigh bone) were inoculated with 103 colony forming units of methicillin-resistant $S$. aureus suspension in group 1 (gram-positive) and E. coli suspension in group 2 (gram-negative). After 7 days, implant-centered infection was established in all animals. Hardtissue histopathology was performed in 2 isolated infected vertebrae from each rabbit. Since increased pressure within the vertebra was suggested as a reason for finding a cold pattern on the labeled WBC scan in proven osteomyelitis, femur infection was induced for comparison as control to assess this theory as opposed to a microbiological characteristic of the causative organisms.

\section{Radionuclide Imaging}

The 32 successfully infected rabbits were used for the rest of the study for imaging. The remaining 18 rabbits were not included in the study due to unsuccessful induction of infection, death, or other technical difficulties. Radionuclide images were obtained for the 2 groups of rabbits 8 days after induction of infection. Each rabbit was intravenously injected with $11.1 \mathrm{MBq}(300 \mu \mathrm{Ci})$ of ${ }^{111} \mathrm{In}-\mathrm{WBC}$. After $24 \mathrm{~h}$, the rabbits were anesthetized and positioned posteriorly. Static images were acquired using gamma camera equipped with a medium-energy and high-resolution collimator interfaced 


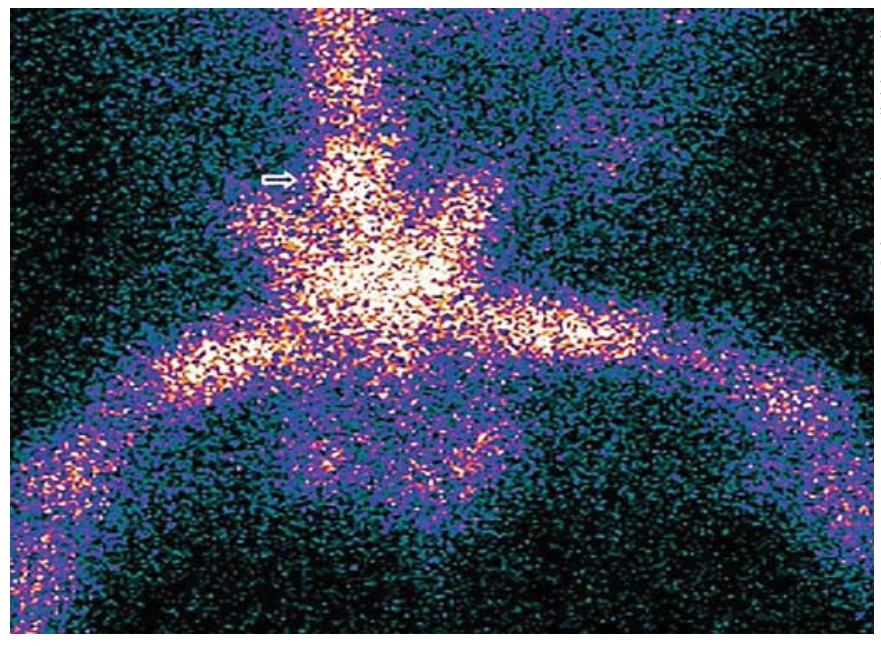

Fig. 1. Posterior image of a rabbit with proven induced gram-positive vertebral osteomyelitis showing accumulation of ${ }^{111}$ In-WBC at the site of infection (arrow).

with a dedicated computer over the area of infection on a matrix of $256 \times 256$. The same imaging protocol was repeated at 14,22 , and 30 days after injection for each rabbit.

\section{Histopathological Procedure}

Two infected vertebrae from each rabbit were isolated and stored in formalin. Decalcification of the bone tissues was done using a decalcification solution. Samples of infected foci in the left femur were also obtained. The samples were dehydrated and then embedded in wax. Using a microtome, 5 - $\mu \mathrm{m}$ sections were cut and mounted on slides. The slides were stained with Gram stain and examined using light microscopy.

\section{Results}

\section{Osteomyelitis Induction}

Histopathological studies confirmed the induction of infection in each rabbit used in the ${ }^{111} \mathrm{In}-\mathrm{WBC}$ imaging. Active infection in the foci of the infected thigh bones was also histologically confirmed in the rabbits used for imaging.

\section{Radionuclide Imaging}

Based on the obtained static images, of the 8 grampositive-infected rabbits, moderate or high ${ }^{111} \mathrm{In}$-WBC accumulation was observed in 7 (87.5\%) lumbar vertebrae (Fig. 1). In addition, of the 8 gram-positive-infected rabbits in the left thigh bone, 7 (87.5\%) showed accumulation in the infected site (Fig. 2). In contrast, in the gramnegative-infected group, 1 (12.5\%) of the 8 infected rab-

${ }^{111}$ In-WBC Scintigraphy in Vertebral Osteomyelitis

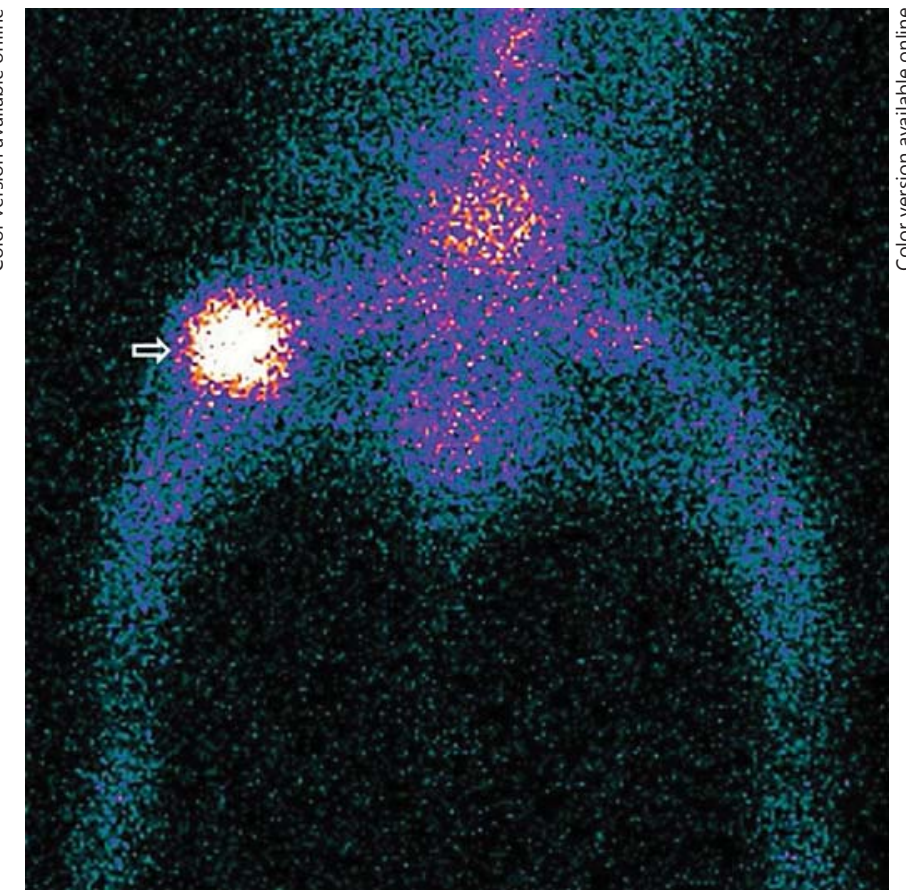

Fig. 2. Posterior image of a rabbit with proven induced left femur infection induced by Staphylococcus aureus (gram-positive) showing high accumulation of ${ }^{111} \mathrm{In}-\mathrm{WBC}$ at the site of infection (arrow).

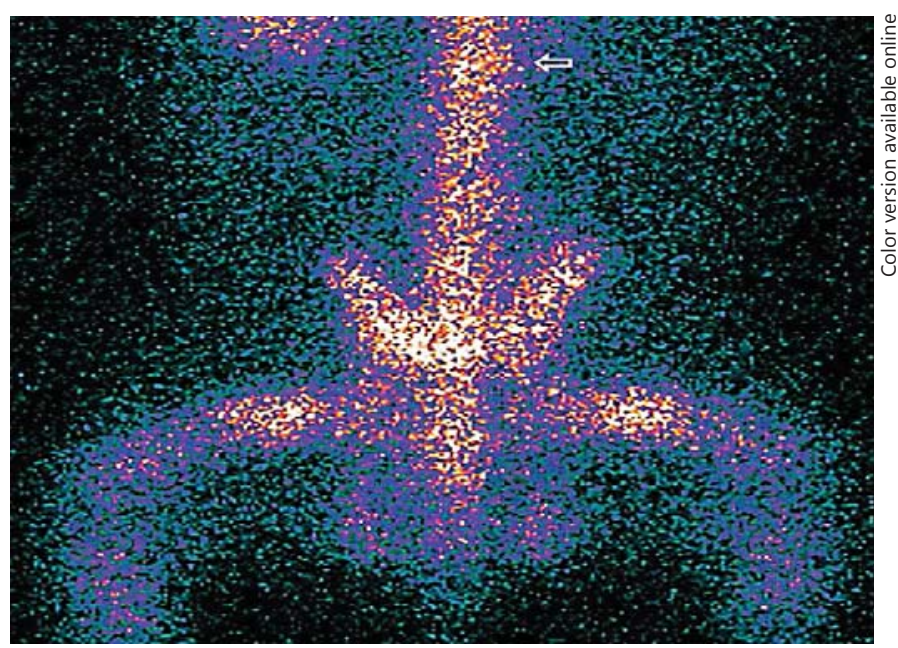

Fig. 3. Posterior image of a rabbit with proven induced gram-negative vertebral osteomyelitis showing minimal accumulation of ${ }^{111}$ In-WBC at the site of infection (arrow).

bits in the vertebrae had little accumulation of ${ }^{111} \mathrm{In}$ WBC, as shown in Figure 3, while the rest had no or decreased accumulation (Fig. 4) at the site of the infected vertebrae. Of the 8 gram-negative-infected rabbits in the

Med Princ Pract 2017;26:415-420 


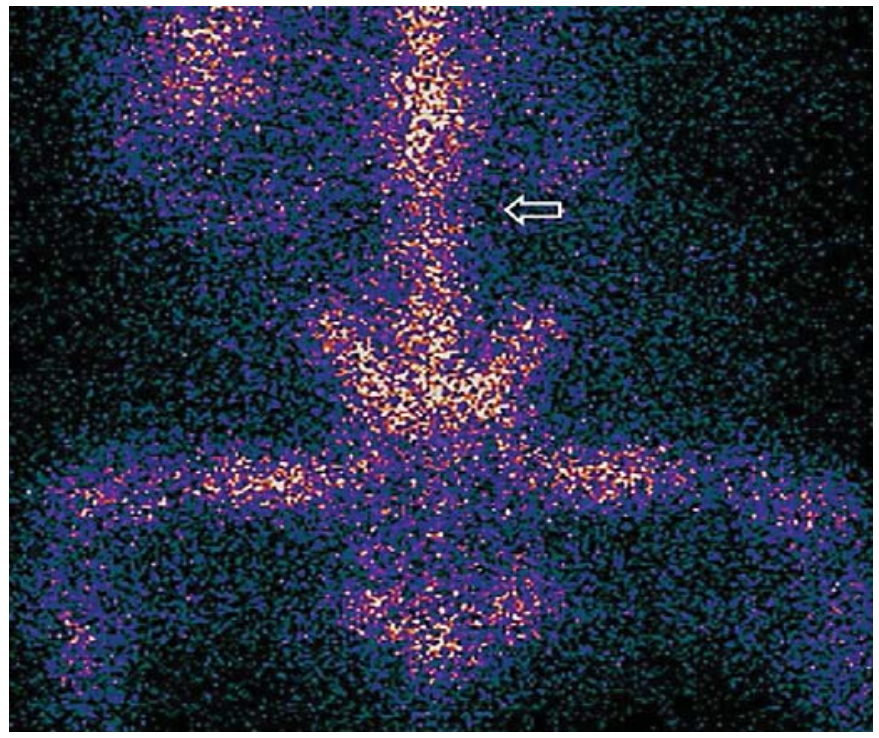

Fig. 4. Posterior image of a rabbit with proven induced gram-negative vertebral osteomyelitis showing decreased accumulation of ${ }^{111} \mathrm{In}-\mathrm{WBC}$ at the site of infection (arrow).

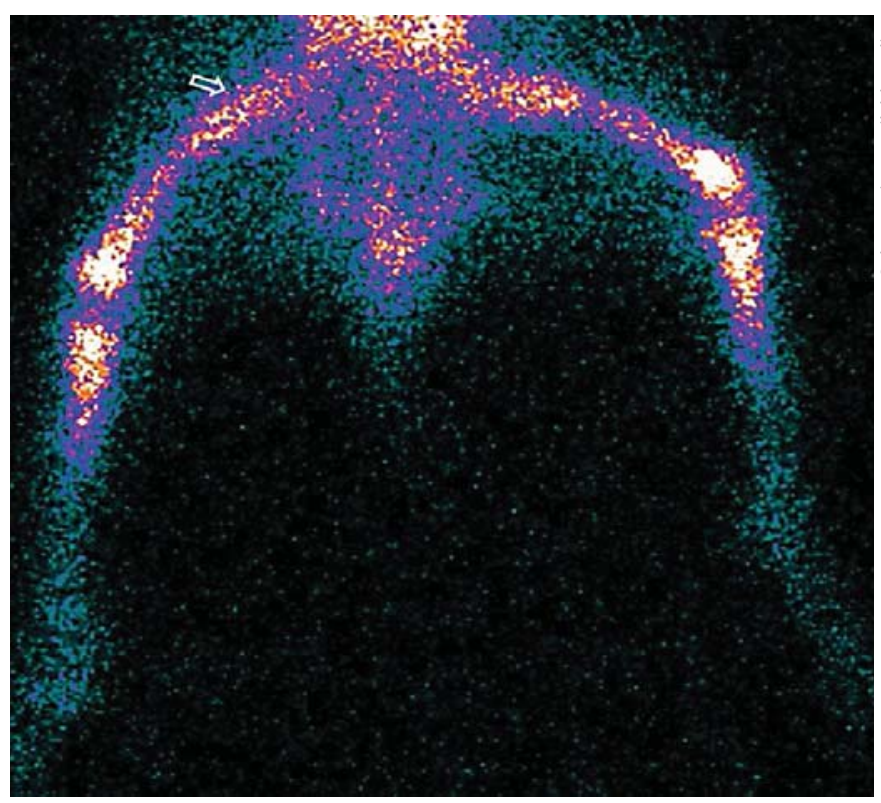

Fig. 5. Posterior image of a rabbit with proven induced gram-negative infection in the left femur showing no accumulation of ${ }^{111} \mathrm{In}-\mathrm{WBC}$ at the site of infection (arrow).

left thigh bone, 6 did not show accumulation of ${ }^{111}$ InWBC at the site of proven infection (Fig. 5), whereas 2 rabbits had accumulation of ${ }^{111}$ In-WBC (25\%) - one with high accumulation (Fig. 6) and the other with low accumulation (Fig. 7).

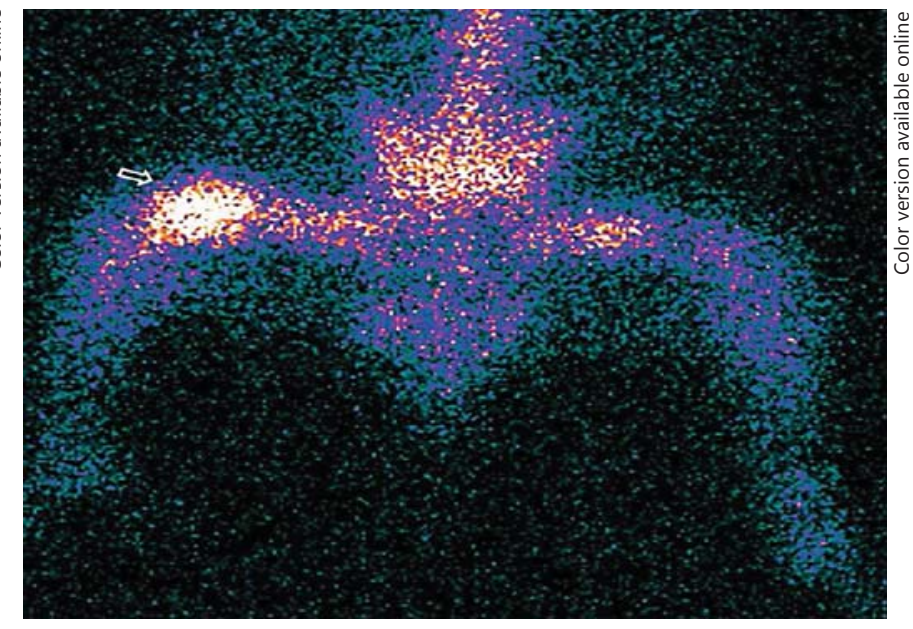

Fig. 6. Posterior image of a rabbit with proven induced gram-negative infection in the left femur showing high accumulation of ${ }^{111}$ In-WBC at the site of infection (arrow).

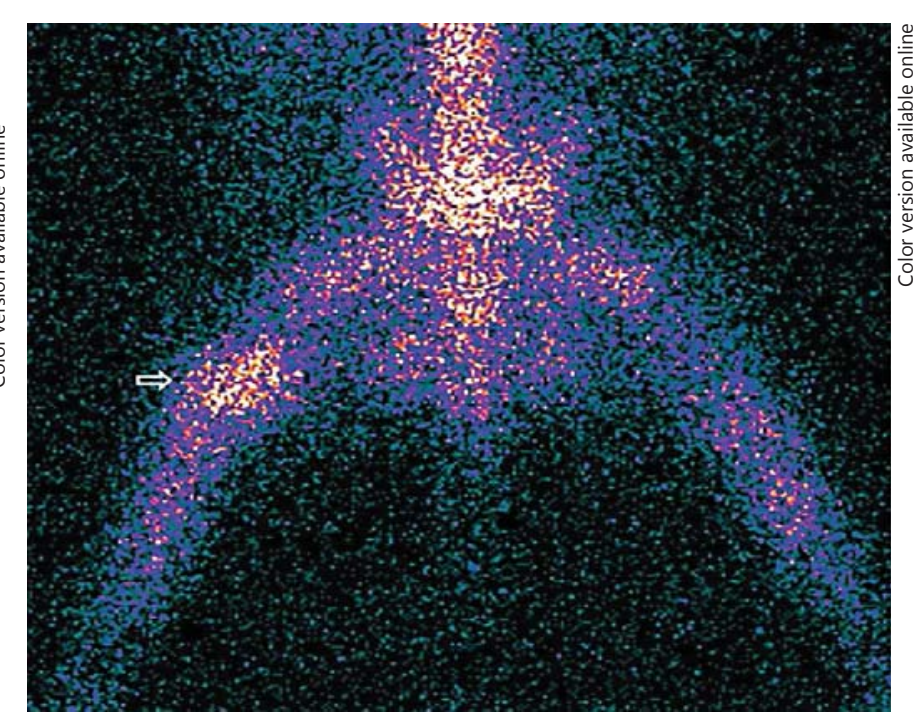

Fig. 7. Posterior image of a rabbit with proven induced gram-negative infection in the left femur showing low accumulation of ${ }^{111}$ In-WBC at the site of infection (arrow).

\section{Discussion}

The current study showed that the majority of infections caused by gram-negative organisms were negative, while the majority of gram-positive infections were positive on ${ }^{111}$ In-WBC-labeled scintigraphic images. The nonaccumulation of ${ }^{111} \mathrm{In}-\mathrm{WBC}$ at the site of infection due to gram-negative organisms confirmed previous findings [4-6], in which proven cases of vertebral osteo- 
myelitis did not show radionuclide accumulation at the site of infection but rather a cold pattern (nonaccumulation). Additionally, cold patterns have also been reported in cases with proven vertebral osteomyelitis using antigranulocyte antibodies [12-16]. The suggested explanation was a compartmental effect due to increased pressure in the infected vertebrae in addition to previous antibiotic therapy and occlusion of microcirculation leading to necrosis [16]. In this study, because the patterns of accumulation or nonaccumulation of ${ }^{111} \mathrm{In}$-WBC in femur infection were similar to those of the vertebrae, it could indicate that the compartment theory with high pressure was not responsible for the cold pattern on the scans in gram-negative infections but rather due to the bacteriological characteristics of the causative organisms.

In 5 cases with proven osteomyelitis, we previously reported a cold pattern in 4 , while the fifth was positive; the 4 cold cases were due to gram-negative organisms, while the positive case was due to gram-positive organisms [12]. An explanation of the nonaccumulation at infection sites caused by gram-negative organisms in this report could be secondary to the secretion of antichemotactic factors by some causative organisms such as E. coli, which prevented adequate accumulation of labeled cells at the site of infection.

The positive finding in the cases with gram-negative vertebral and femur infections could probably be due to the degree of severity of infection and, consequently, the amount of antichemotactic factors secreted by the organisms. Thus, if the infection was not severe, the amount of antichemotactic chemicals was not enough to prevent adequate accumulation of labeled WBC to show on the scan.
The negative finding of 1 case with gram-positive organisms could again be due to milder infections without adequate accumulation of the labeled WBC. This negative finding in proven osteomyelitis secondary to gram-negative organisms has been reported in human studies [4-6].

Although osteomyelitis is usually caused by gram-positive organisms, the most common being $S$. aureus, gramnegative organisms such as $P$. aeruginosa and K. pneumonia have been shown to be the causative organisms in osteomyelitis, particularly in intravenous drug abusers, vertebral osteomyelitis, and hospital-acquired infection $[17,18]$. E. coli is also a cause of vertebral osteomyelitis following urological surgery. The gram-negative organisms are known to secrete antichemotactic factors contrary to gram-positive organisms [19-21].

The results of this study indicate that the reason for negative findings was due to the gram-negative causative organisms which are known to secrete antichemotactic factors, preventing adequate accumulation of leukocytes at the site of infection and thus negating visualization on scans.

\section{Conclusion}

In the majority of the gram-positive-infected vertebrae in rabbits there was increased ${ }^{111} \mathrm{In}-\mathrm{WBC}$ accumulation. However, in most of the gram-negative-infected vertebrae no ${ }^{111} \mathrm{In}-\mathrm{WBC}$ accumulation was seen. The results provide support for our hypothesis - to explain for the first time the theory behind finding normal and particularly photon-deficient areas at the sites of vertebral infections on WBC scans.

\section{References}

1 Tyrrell PN, Cassar-Pullicino VN, McCall IW: Spinal infection. Eur Radiol 1999;9;10661077.

2 Lee YJ, Sadigh S, Mankad K, et al: The imaging of osteomyelitis. Quant Imaging Med Surg 2016;6:184-198.

3 Schauwecker DS: Osteomyelitis: diagnosis with In-111-labeled leukocytes. Radiology 1989;171:141-146.

4 Mok YP, Carney WH, Fernandez-Ulloa M: Skeletal photopenic lesions in In-111 WBC imaging. J Nucl Med 1984;25:1322-1326.

5 Datz FL, Thorne DA: Cause and significance of cold bone defects on indium-111-labeled leukocyte imaging. J Nucl Med 1987;28:820823.

${ }^{111}$ In-WBC Scintigraphy in Vertebral Osteomyelitis
6 Hotze AL, Briele B, Overbeck B, et al: Technetium-99m-labeled anti-granulocyte antibodies in suspected bone infections. J Nucl Med 1992;33:526-531.

7 Palestro CJ, Kim CK, Swyer AJ, et al: Radionuclide diagnosis of vertebral osteomyelitis: indium-111-WBC and technetium-99mmethylene diphosphonate bone scintigraphy. J Nucl Med 1991;32:1861-1865.

8 Whalen JL, Brown ML, McLeod R, et al: Limitations of indium leukocyte imaging for the diagnosis of spine infections. Spine 1991;16: 193-197.
9 Rüther W, Hotze A, Möller F, et al: Diagnosis of bone and joint infection by leucocyte scintigraphy. A comparative study with $99 \mathrm{mTc}$ HMPAO-labelled leucocytes, $99 \mathrm{mTc}$-labelled antigranulocyte antibodies and $99 \mathrm{mTc}-\mathrm{la}-$ belled nanocolloid. Arch Orthop Trauma Surg 1990;110:26-32.

10 Hovi I: Complicated bone and soft-tissue infections. Imaging with $0.1 \mathrm{~T}$ MR and $99 \mathrm{mTc}$ HMPAO-labeled leukocytes. Acta Radiol 1996;37:870-876

11 Lazzeri E, Pauwels EKJ, Erba PA, et al: Clinical feasibility of two-step streptavidin/111Inbiotin scintigraphy in patients with suspected vertebral osteomyelitis. Eur J Nucl Med Mol Imaging 2004;31:1505-1511. 
12 Fernandez-Ulloa M, Vasavada PJ, Hanslits ML, et al: Diagnosis of vertebral osteomyelitis: clinical, radiological and scintigraphic features. Orthopedics 1985;8:1144-1150.

13 Poelstra KA, Barekzi NA, Grainger DW, et al: A novel spinal implant infection model in rabbits. Spine 2000;25:406-410.

14 Palestro CJ: Radionuclide imaging of osteomyelitis. Semin Nucl Med 2015;45:32-46.

15 Elgazzar AH, Abdel-Dayem HM, Clark JD, et al: Multimodality imaging of osteomyelitis. Eur J Nucl Med 1995;22:1043-1063.
16 Gratz S, Braun HG, Behr TM, et al: Photopenia in chronic vertebral osteomyelitis with technetium-99m-antigranulocyte antibody (BW 250/183). J Nucl Med 1997;38:211-216.

17 Waldvogel FA, Medoff G. Swartz MM: Osteomyelitis: a review of clinical features, therapeutic considerations and unusual aspects. $\mathrm{N}$ Engl J Med 1970;282:198-206.

18 Kahn DS, Pritzker KPH: The pathophysiology of bone infection. Clin Orthop Relat Res 1973;96:12-19.
19 Kumar JV, Abbas AK, Astor JC: Inflammation and repair; in Kumar JV, Abbas AK, Astor JC (eds): Robins and Cotran Pathologic Basis of Disease, ed 9. Philadelphia, ElsevierSaunders, 2015, pp 69-112.

20 Torda AJ, Gottlieb T, Bradbury R: Pyogenic vertebral osteomyelitis: analysis of 20 cases and review. Clin Infect Dis 1995;20:320-328.

21 Song KS, Ogden JA, Ganey T, et al: Contiguous discitis and osteomyelitis in children. J Pediatr Orthop 1997;17:470-477. 